\title{
Comparing Realistic Subthalamic Nucleus Neuron Models
}

Felix Njap, Jens C. Claussen, Andreas Moser, and Ulrich G. Hofmann

Citation: AIP Conference Proceedings 1371, 102 (2011); doi: 10.1063/1.3596632 View online: https://doi.org/10.1063/1.3596632

View Table of Contents: http://aip.scitation.org/toc/apc/1371/1

Published by the American Institute of Physics

\section{Conference Proceedings}

Get $30 \%$ off all

Enter Promotion Code PDF-30 a print proceedings! 


\title{
Comparing Realistic Subthalamic Nucleus Neuron Models
}

\author{
Felix Njap $^{\mathrm{ab}}$, Jens C. Claussen ${ }^{\mathrm{cb}}$, Andreas Moser ${ }^{\mathrm{d}}$, Ulrich G. Hofmann ${ }^{\mathrm{a}}$

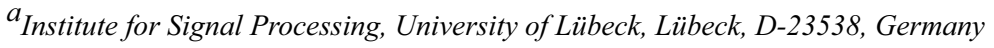 \\ ${ }^{b}$ Graduate School for Computing in Medicine and Life Sciences, University of Lübeck, D-23538, Germany \\ ${ }^{c}$ Institute for Neuro-and Bioinformatics, University of Lübeck, Lübeck, D-23538, Germany

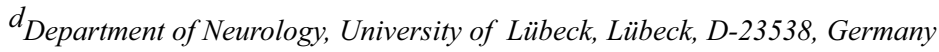

\begin{abstract}
The mechanism of action of clinically effective electrical high frequency stimulation is still under debate. However, recent evidence points at the specific activation of GABA-ergic ion channels. Using a computational approach, we analyze temporal properties of the spike trains emitted by biologically realistic neurons of the subthalamic nucleus (STN) as a function of GABA-ergic synaptic input conductances. Our contribution is based on a model proposed by Rubin and Terman and exhibits a wide variety of different firing patterns, silent, low spiking, moderate spiking and intense spiking activity. We observed that most of the cells in our network turn to silent mode when we increase the GABAA input conductance above the threshold of $3.75 \mathrm{mS} / \mathrm{cm}^{2}$. On the other hand, insignificant changes in firing activity are observed when the input conductance is low or close to zero. We thus reproduce Rubin's model with vanishing synaptic conductances. To quantitatively compare spike trains from the original model with the modified model at different conductance levels, we apply four different (dis)similarity measures between them. We observe that Mahalanobis distance, Victor-Purpura metric, and Interspike Interval distribution are sensitive to different firing regimes, whereas Mutual Information seems undiscriminative for these functional changes.
\end{abstract}

Keywords: $\gamma$-Aminobutyric acid (GABAA) receptors; spike-trains; similarity measures; computational model. PACS: $01.30 . C c$

\section{INTRODUCTION}

Deep brain stimulation (DBS) of subthalamic nucleus (STN) by means of implanted electrodes has become a well accepted technique for treatment of several movement disorders $[1,2,3]$. However, the mechanisms of the molecular and pharmacological action of periodic electrical high frequency stimulation (HFS, $\mathrm{f}>100 \mathrm{~Hz}$ ) are still under dispute $[4,5,6,7]$.

To understand the network and cellular mechanisms responsible for the efficacy of HFS, several attempts have been made to mathematically model diseases related to movement disorders and other neurological disorders [8,9,10]. In 2004 Rubin and Terman [11] proposed a mechanism for HFS in Parkinson's disease (PD) based on single-compartment biophysically realistic Hodgkin-Huxley $(\mathrm{HH})$ models for five interconnected subcortical nuclei. Reported firing pattern modifications in the simulated network have been intensively studied and simulated in other computer models using different approaches [12].

Since it was proposed that the GABA system is involved in the effectiveness of DBS [ 4,5,6,7], our intention is to investigate this mechanism in a modified version of Rubin's STN model. In detail, we added postsynaptic, GABArelated currents to the compartment receiving inhibitory stimulation-induced trans-synaptic input. Clearly, with this step we are leaving the bases of experimentally available network data and proceed on other paths to validate our model. Therefore, the current study seeks to numerically examine the extend to which both spiking models are similar or have different behavior as the synaptic input conductance fluctuates. Since both models produce spike train output, we apply different spike similarity measures to compare both biologically realistic subthalamic nucleus (STN) neuron models. Synaptic input conductances consist of two sets of ionic synaptic conductances (small and large) for action potential or spike generation.

We introduced an additional stochastic component, i.e noise, to our models so that not only different instantiations of the model are different from each other, but also to obtain more realistic simulations. Such noisy patterns are typical for many neurons in the brain [13]. Due to different ions concentration levels, the model produces irregular burst activity that can be observed during resting conditions in the basal ganglia. In the next section, we will describe the equations that define our models. 


\section{MATHEMATICAL MODEL}

The basic model used in this paper is identical to the one described in [11]. It consists of five anatomical nuclei representing the external segment of globus pallidus (GPe), the subthalamic nucleus (STN), the internal segment of globus pallidus (GPi), thalamus, and cortex, where the first three nuclei belong to the basal ganglia network. We modified the STN cells in such a way as to display the possible effects of neurotransmitter GABA channels. Therefore, we used the following $\mathrm{HH}$ model to mathematically describe the membrane potential $\mathrm{V}$ of singlecompartment STN neurons, given by the equation (1):

$$
C_{m} \frac{d V}{d t}=-I_{N a}-I_{k}-I_{C a}-I_{T}-I_{A H P}-I_{L e a k}-I_{S y n}+C_{m} \eta
$$

where $C_{m}$ is the membrane capacitance. Incorporated ionic currents are described as follows:

$\mathrm{Na}^{+}$current with instantaneous activation variable $m_{\infty}$ and inactivation variable $\mathrm{h} ; I_{\mathrm{Na}}=g_{\mathrm{Na}} m_{\infty}^{3} h\left(V-E_{\mathrm{Na}}\right)$.

$K^{+}$delayed rectifier current, (high activation threshold, fast activation time constant), with activation variable n; $I_{K}=g_{K} n^{4}\left(V-E_{K}\right)$.

$\mathrm{Ca}^{2+}$ high-threshold current with instantaneous activation variable $s_{\infty} ; I_{C a}=g_{C a} s_{\infty}^{2}\left(V-E_{C a}\right)$.

$\mathrm{Ca}^{2+}$ low-threshold t-type current with instantaneous activation variable $a_{\infty}$ and inactivation variable $b_{\infty}$, $I_{T}=g_{T} a_{\infty}^{3} b_{\infty}^{2}\left(V-E_{T}\right)$.

By using this equation, T-type currents include the effects of a hyper-polarization activated inward current. $\mathrm{Ca}^{2+}$ activated voltage independent after hyper polarization $\mathrm{K}^{+}$current, with $\left[\mathrm{Ca}^{2+}\right]_{i n}$ the intracellular concentration of $\mathrm{Ca}^{2+}$ ions, and $k_{1}$ the dissociation constant of this current $I_{A H P}=g_{A H P}\left(V-E_{K}\right) \frac{\left[\mathrm{Ca}^{2+}\right]}{\left[\mathrm{Ca}^{2+}\right]+k_{1}}$. Leak current $I_{\text {Leak }}=g_{\text {Leak }}\left(V-E_{\text {Leak }}\right)$.

The gating kinetics of the ionic conductances were calculated using the following equation:

$\frac{d n}{d t}=\frac{n_{\infty}-n}{\tau_{n}} ; \quad \frac{d m}{d t}=\frac{m_{\infty}-m}{\tau_{m}} ; \quad \frac{d h}{d t}=\frac{h_{\infty}-h}{\tau_{h}} ;$

$n_{\infty}=\frac{\alpha_{n}}{\alpha_{n}+\beta_{n}} ; \quad m_{\infty}=\frac{\alpha_{m}}{\alpha_{m}+\beta_{m}} ; \quad h_{\infty}=\frac{\alpha_{h}}{\alpha_{h}+\beta_{h}} ;$

$\tau_{n}=\frac{1}{\alpha_{n}+\beta_{n}} ; \quad \tau_{m}=\frac{1}{\alpha_{m}+\beta_{m}} ; \quad \tau_{h}=\frac{1}{\alpha_{h}+\beta_{h}} ;$

The additional $I_{s y n}$ are inhibitory postsynaptic GABA-related currents which can be accessed both by $G A B A_{A}$ and $G A B A_{B}$ subtypes of GABA receptors. In this initial study, only conductance setup of $G A B A_{A}$ is included. This conductance is modeled with the first order kinetic of the binding transmitter to postsynaptic receptors [14].

$\frac{d s}{d t}=\alpha C(1-s)-\beta s$, where $\mathrm{C}$ is the concentration of neurotransmitter in the synaptic cleft given by: $[C]=C_{\max } /\left(1+\exp \left(V-V_{p}\right) / k_{p}\right), \alpha$ and $\beta$ are the forward and backward rate constants, and s represents the fraction of open channels on the postsynaptic membrane. The postsynaptic current receiving inhibitory stimulation input is given by:

$I_{G A B A_{A}}=g_{G A B A_{A}} s_{G A B A_{A}}\left(V_{m}-E_{G A B A_{A}}\right)$; where $V_{m}$ is the postsynaptic membrane potential, $g_{G A B A_{A}}$ is the peak conductance and $E_{G A B A_{A}}$ is the reversal potential. $C_{m} \eta$ is the stochastic noise current component added to our models. Simulations were performed using XPPAUT as platform developed by Bard Ermentrout and MATLAB for analysis. 
The numerical method used for integration was an adaptive step fourth-order Runge-Kutta method with a maximum step width of $0.01 \mathrm{~ms}$.

Parameters describing synaptic current and membrane potential are listed in Table 1.

\begin{tabular}{|c|c|c|c|}
\hline Parameters & $\alpha, m M^{-1} m s^{-1}$ & $\beta, m s^{-1}$ & $\mathrm{E}, \mathrm{mV}$ \\
\hline$\overline{G A B A_{A}}$ & 0.53 & 0.184 & $-85 \mathrm{mV}$ \\
\hline Threshold & & & $-45 \mathrm{mV}$ \\
\hline$V_{p}$ & - & - & $0 \mathrm{mV}$ \\
\hline Cmax & - & - & 1 \\
\hline
\end{tabular}

\section{METHODS}

Since the basic problem we are facing here is the question on how similar patterns of stochastically firing cells are, we will in this section briefly describe different similarity measures. We observed simulated neuronal activity of individual cells for each realistic model for a time duration of $1000 \mathrm{msec}$. The sequence of time occurrence of spikes $\left\{t_{0}, t_{1}, \ldots, t_{n}\right\}$,where $0 \leq t_{0}<t_{1}<\ldots<t_{n}<T$

is called a spike-train. In this contribution pairs of spike trains are compared.

\subsection{Mahalanobis Distance}

The Mahalanobis distance is described in [15]. It is based on correlations between variables by which different patterns can be identified and analyzed. The Mahalanobis distance can be defined as a dissimilarity measure between two random vectors $\vec{x}$ and $\vec{y}$ of the same distribution with the covariance matrix $\mathrm{S}$ :

$$
d(\vec{x}, \vec{y})=\sqrt{(\vec{x}-\vec{y})^{T} S^{-1}(\vec{x}-\vec{y})} .
$$

If the covariance matrix $\mathrm{S}$ is the identity matrix (respectively diagonal), then the Mahalanobis distance is reduced to the Euclidean distance respectively (normalized Euclidean distance).

\subsection{Mutual Information}

Mutual information [16,17] relies on an estimation of entropy which measures the degree of uncertainty of a random variable and thereby measures how much information can be encoded with it. The entropy $\mathrm{H}(\mathrm{X})$ of a discrete $\mathrm{X}$ random variable taking the values $a \in \chi$ with probabilities $p(a)=\operatorname{Pr}\{X=a\}$ is defined as:

$$
H(X)=-\sum_{a \in \chi} p(a) \log _{2} p(a)
$$

and is measured in bits. Mutual information for two random variables $\mathrm{X}$ and $\mathrm{Y}$ can be defined as a quantity that measures the mutual dependence of two variables. Mathematically expressed as:

$$
I(X, Y)=H(X)+H(Y)-H(X, Y)
$$

where $\mathrm{H}(\mathrm{X})$ and $\mathrm{H}(\mathrm{Y})$ are marginal entropies, and $\mathrm{H}(\mathrm{X}, \mathrm{Y})$ is their joint entropy. Using the definition of entropy $\mathrm{H}$ $(\mathrm{X})$ and joint entropy $\mathrm{H}(\mathrm{X}, \mathrm{Y})$ the mutual information can be equivalently expressed as:

$$
I(X, Y)=H(X)-H(X / Y)
$$

where $H(X / Y)$ is the conditional entropy.

In the case of spike-trains, time is discretized into bins. Each bin has the value of 1 if a spike occurred within its time duration, otherwise its value is 0 . This process is treated as stochastic with given probability for each of these two possible events. The resulting binary string represents the spike trains and is used for entropy estimation. 
Entropy and mutual information are measured in bits. One significant drawback of mutual information and entropy is that both can not be estimated from correlation data, since they depend on the underlying probability distributions.

\subsection{InterSpike Interval Distance}

The Interspike Interval Distance measure is described in [18]. It can be defined as a time-resolved measure of the firing rate of the spike train or the time between subsequent action potentials. The mean value of the ISI is calculated according to (6)

$$
\overline{I S I}=\frac{1}{n} \sum_{i=i}^{n} I S I_{i}
$$

\subsection{Victor-Purpura Distance Metric}

The Victor-Purpura spike metric was described in[19]. It defines the distance between two spike trains as the minimum cost of transforming one spike train into the other. It combines three basic operations: spike insertion, spike deletion and spike movement. The cost $D v$ of inserting or deleting a spike is set to one, the cost of moving a spike in time is proportional to the time shift. The time scale of the distance metric is defined by the proportionality constant $\mathrm{Cv}$. If $C v$ is equal to zero, the distance metric $D v$ basically equals to the difference in spike number, whereas for large $C v$, the distance approaches the number of non-coincident spikes, since instead of shifting spikes it becomes more favorable to delete all non-coincident spikes of the one time series and to insert non-coincident spikes of the other. Increasing $C v$, the distance is transformed from a rate distance to a timing distance.

All the above similarity measures have advantages and disadvantages. They were extensively discussed and successfully implemented as analyzing tools for many spike trains in $[20,21,22]$.

\section{SPIKE DETECTION}

Spike detection was done by thresholding of simulated extracellular [23] potential. We determined the threshold voltage as the average of the maximum peak value and the minimum peak value of action potentials. As soon as the simulated voltage exceeds the determined threshold a spike is emitted and the depolarization is reset. This method is by far the most common for measuring neural activity. It has an advantage that it requires minimal hardware and software, and can obtain exactly desired information. On the other hand it is not always possible to achieve acceptable discrimination

\section{RESULTS}

\subsection{Firing Pattern}

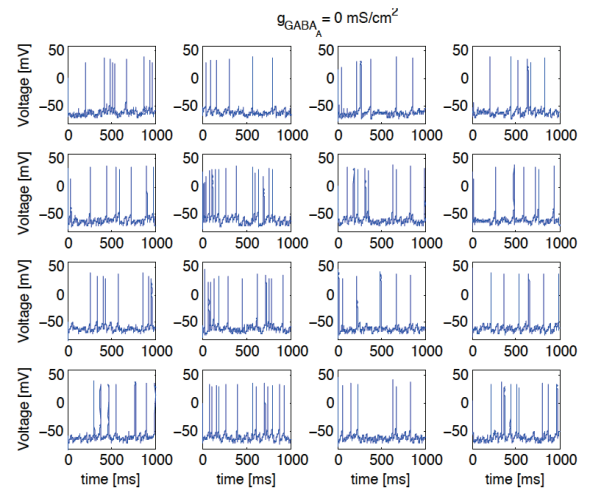

(a)

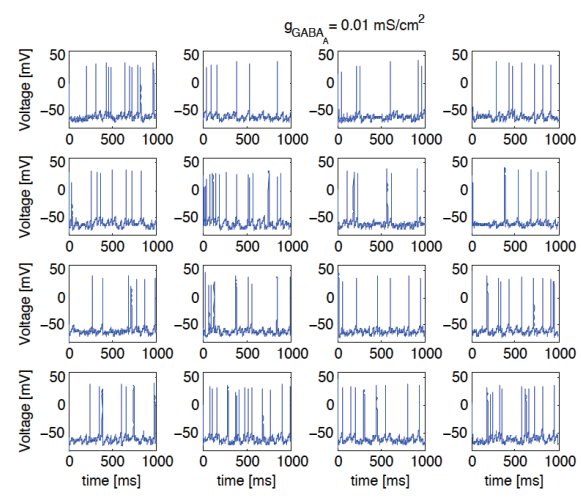

(b) 


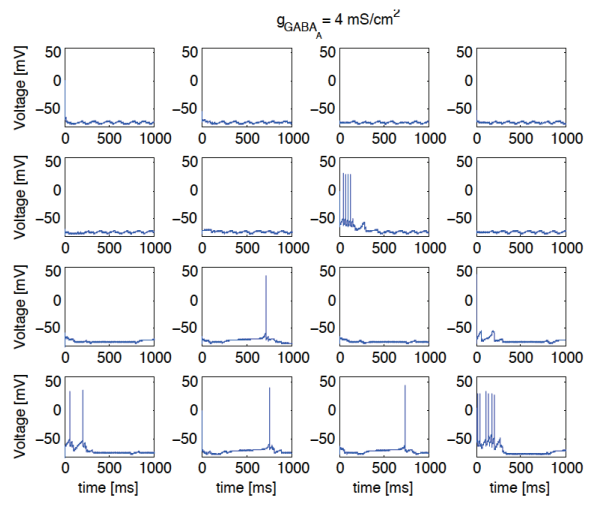

(c)

FIGURE 1. Various firing patterns exhibited by the model. Each voltage trace represents one of sixteen neurons in the STN model. Traces were simulated by using weaker or larger synaptic conductances, 0 (Fig.1a), 0.01(Fig.1b) and $4 \mathrm{mS} / \mathrm{cm}^{2}$ (Fig.1c) respectively. Four firing types can be observed. The spiking activity changes dramatically, when the strength of local inhibition by the higher GABA conductance was increased.

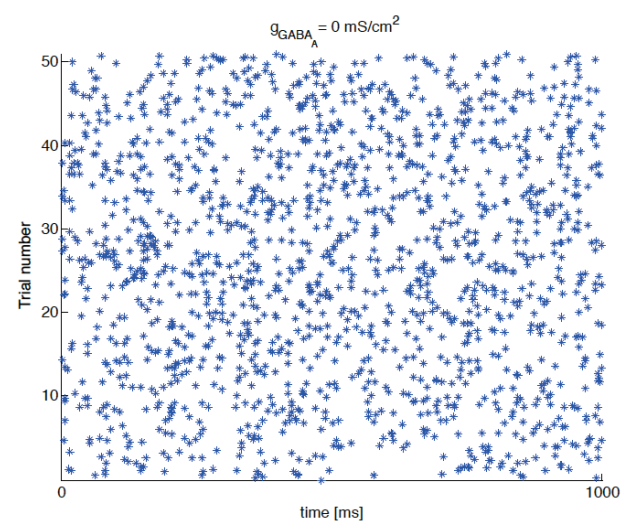

(a)

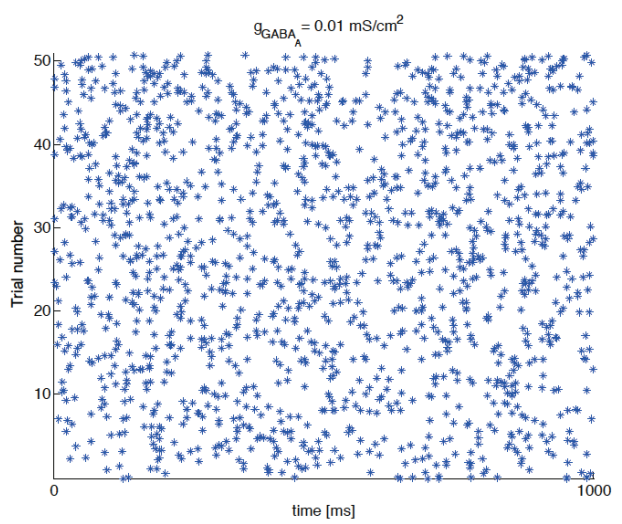

(b)

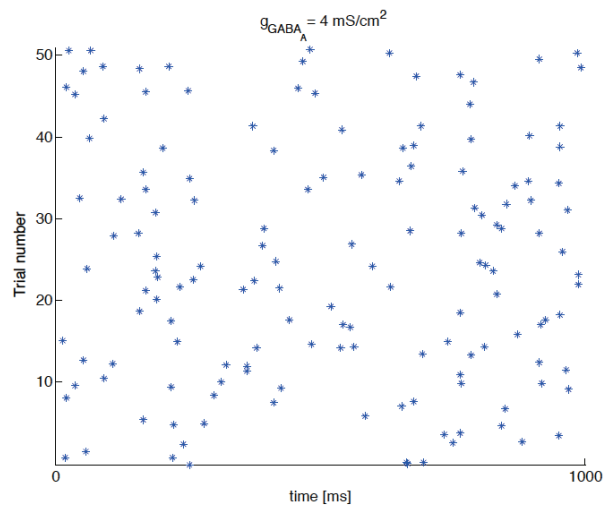

(c) 
FIGURE 2. Rastergram for one cell inhibited by a synaptic conductance $0 \mathrm{mS} / \mathrm{cm}^{2}$ (top left) $0.01 \mathrm{mS} / \mathrm{cm}^{2}$ (top right) and $4 \mathrm{mS} /$ $\mathrm{cm}^{2}$ (bottom). Spikes seem to be stochastically distributed.

We begin our simulation using two different input regimes consisting of smaller and larger synaptic conductance input. We start with $g_{G A B A=0}$, which fully reproduced Rubin's model and gradually increased the synaptic conductances with step size $0.01 \mathrm{mS} / \mathrm{cm}^{2}$ up to $5 \mathrm{mS} / \mathrm{cm}^{2}$. The basic bursting behavior is shown in Fig $1 \mathrm{a}, 1 \mathrm{~b}, 1 \mathrm{c}$.

By thresholding the membrane potential V, we obtain the rastergram in Fig 2. Here we display 50 trials of 1000 msec lengths for the input conductance $0 \mathrm{mS} / \mathrm{cm}^{2} 0.01 \mathrm{mS} / \mathrm{cm}^{2}$ and 50 trials for $4 \mathrm{mS} / \mathrm{cm}^{2}$.

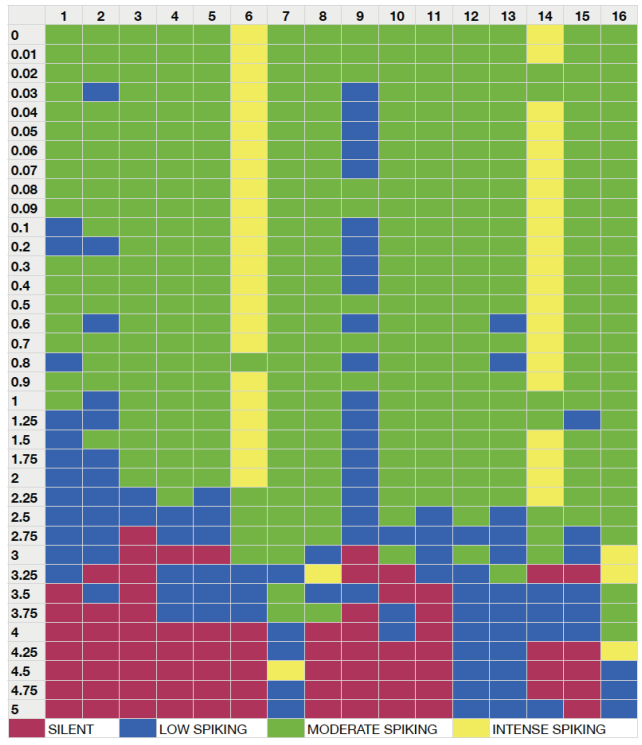

FIGURE 3 Firing types corresponding to different synaptic conductances input. Each row represents the synaptic conductances input. Each column represents an exemplary STN's cell number.Each color entry denotes the qualitative electrophysiological response of a particular cell to a level of synaptic conductance input.

All our simulations showed STN neurons differ in their firing pattern depending on the synaptic parameter (GABA) strength. For large conductance inputs, this fluctuation can have very big effects on the rate of variability of firing of individual neurons. These effects depend strongly on the synaptic connectivity and neuronal mechanism. In particular, we observed four activity regimes:

Silent dominated regime mostly when the value of synaptic conductance input is above 4 , in this regime no spiking activity was observed;

Low spiking activity regime characterized by less than 10 spikes per second;

Moderate spiking activity regime where the number of spikes per second lies between 10 and 25;

Intense spiking activity regime where the number of spikes per second observed exceeded 25 .

In all our simulations, the firing pattern was mostly irregular as observed in the normal state. The analysis of qualitative firing involved taking all cells and organizing them into these four firing types.

By categorizing the cell's outputs based on firing rate and comparing them with the amount of inhibition in our model, Fig. 3 shows that almost no inhibition

occurs up to conductances of $0.9 \mathrm{mS} / \mathrm{cm}^{2}$. The neurons behave similar to the original Rubin model.

However, this qualitative picture has to be investigated more carefully, since with the increasing synaptic input conductances, the mutual information of the model almost vanishes and Mahalanobis distance metric start increasing despite seemingly comparable qualitative behavior. 


\subsection{Similarity Measures}

Tests of above described similarity measures were conducted on each of the sixteen STN cells. For each synaptic conductance input, we produced several ( $\mathrm{n}=50$ trials) independently simulated spike trains, and calculated from them the Mahalanobis distance (Mah Dist), the Victor-Purpura distance metric (Vic Dist), the Mutual Information $\left(M u_{-}\right.$Info) to the Rubin's model and the Interspike interval distance

(ISI_Dist) as the average sum of all different trials for each different cell number. Table 2 gives normalized average value for four metrics based on their spiketimes recorded.

TABLE 2. Normalized metrics for four different synaptic

\begin{tabular}{llcccc}
\multicolumn{6}{l}{ conductances input. } \\
\hline$g_{G A B A_{A}}$ & 0 & 0.01 & 0.1 & 1 & 5 \\
\hline Mah_Dist & 0 & 0.07 & 0.101 & 0.130 & 0.997 \\
Vic_Dist & 0 & 0.742 & 0.825 & 0.904 & 0.512 \\
ISI_Dist & 0 & 0.457 & 0.393 & 0.539 & 0.999 \\
Mu_Info & 1 & 0.1 & 0.05 & 0.015 & 0.015 \\
\hline
\end{tabular}

Increasing synaptic input conductances, the Mutual information of the model's output rapidly vanishes, the Mahalanobis distance metric, the Interspike Distance and the Victor-Purpura distance starts to increase (Fig. 4). This contradicts the seemingly smooth transition from the Rubin model cell $\left(g_{G A B A}=0\right)$ to our model as seen in the spike rate picture. However, up to an input conductance of $1,75 \mathrm{mS} / \mathrm{cm}^{2}$, the further increase in all these values is quite slow, corroborating a common regime governed by the GABA-conductance. And yet, the spike rate activity here remains quite stable as seen in Fig. 3. Above $1,75 \mathrm{mS} / \mathrm{cm}^{2}$ all similarity measures start to drastically change their values and, as can be seen in Fig.3, the spiking activity becomes less homogeneous until most cells turn silent at values above $3,75 \mathrm{mS} / \mathrm{cm}^{2}$.

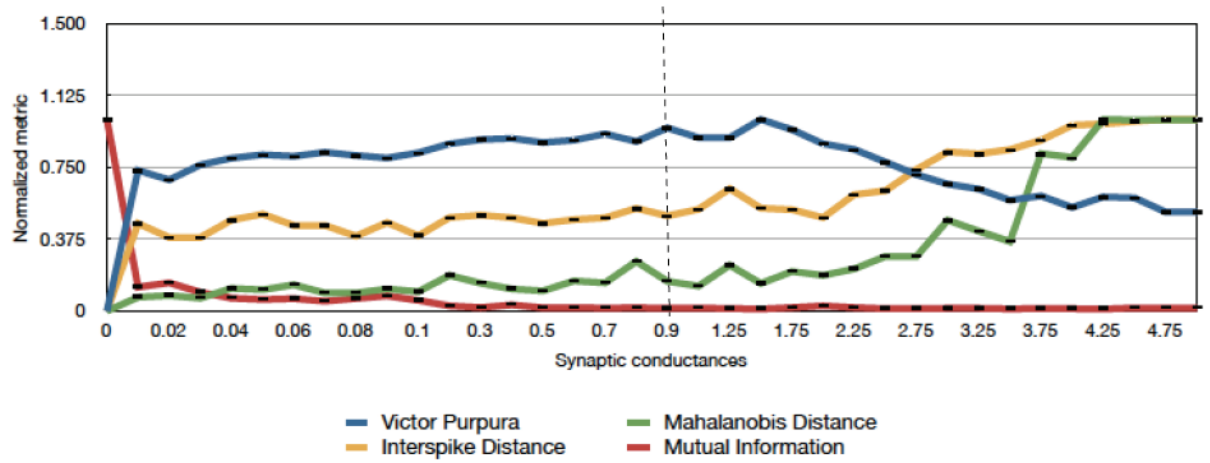

FIGURE 4. Comparison of different similarity measures for increasing synaptic input. The dashed lines represents threshold where our model behave similar to Rubin's model.

\section{DISCUSSIONS AND CONCLUSION}

Our original intention was to improve an existing basal ganglia model by a GABA-mediated synaptic conductance to investigate its relevance as potential target of electrical high frequency stimulation.

Not unexpectedly, the additional synaptic current $g_{G A B A}$ has a significant effect on the model neurons electrophysiological response. GABAA receptors are located on glutamatergic neurons, thus these neurons would be inhibited by increased GABA activity.

In order to validate this model, we had to find a way to compare our model's output to the existing model and experimental data. But how to compare stochastically produced spike trains from one model to another? So the question dealt with in this study, was to utilize existing similarity methods to monitor increasing discrepancies between the base model and ours by increasing synaptic input conductances. 
We found out, that besides the mutual information all three metrics are useful to detect differences in different firing regimes. Opposite to that, the standard spike rate view was to insensitive to determine a clear transition in output behavior of the model, not of one single particular cell.

All in all we are now confident that our model does not violate the assumptions found in the basic basal ganglia model of Rubin, but whether or not it will provide an alternative mechanism of action of high frequency stimulation (HFS) is subject to further investigations.

\section{ACKNOWLEDGMENTS}

This work was supported by the "Graduate School for Computing in Medicine and Life Sciences" funded by Germany`s Excellence Initiative [DFGGSC235/1].

\section{REFERENCES}

1. A. Benabid, A. Benazzouz and P. Pollak, Mov. Disorders 17, 73-74 (2002).

2. A. Benabid, Expert Rev. Med. Devices 4, 895-903 (2007).

3. C.C McIntyre, M. Savasta, L. B. Walter and J.L. Vitek, J. Clin. Neurophys. 21, 40-50 (2004).

4. A. Moser, A. Gieselberg, B. Ro, C. Keller and F. Qadri, Neurosci. Letters 341, 57-60 (2003).

5. M. Mantovani, A. Moser, C.A.Haas, J. Zentner and T. J. Feuerstein, Naunyn-Schmiedberg's Arch Pharmacol 380, 45-58 (2009).

6. A. Hiller, S. Loeffler, C. Haupt, M. Litza, U. Hofmann and A. Moser, J. Neurosci. Meth. 159, $286-290$ (2007).

7. M. Mantovani, V. van Velthoven, H. Fuellgraf, T.J. Feuerstein and A. Moser, Neurochem. Int. 49, 347-350 (2006).

8. .J.E. Arle, L.Z. Mei and J.L. Shils, Stereotact. Funct. Neurosurg. 86, 1-15 (2008).

9. J.E. Arle, L.Z. Mei, J.L. Shils, Stereotact. Funct. Neurosurg. 86, 16-29 (2008).

10. M. Flint Beal, Nature Reviews Neurosci. 2, 325-334(2001).

11. J. E. Rubin and D. Terman, J. Comput. Neurosci. 16, 211-223 (2004).

12. T.R. Chay, F.S. Fan and F.S. Lee, Int. J. Bifur. Chaos 5, 595-635 (1995).

13. M. Zhang, P. Goforth, R. Bertram, A. Sherman and L. Satin, Biophys. J. 84, 2852-2870 (2003).

14. A. Destexhe, Z.F. Mainen and T.J. Sejnowski, J. Comput. Neurosci. 6, 14-18 (1994).

15. S. Rebrik, B. Wright, A. Emondi and K. D Miller, Neurocomputing 26-27, 1033-1038 (1999).

16. S. Guiasu, Information Theory with Applications, New York: McGraw-Hill, 1977.

17. A. Papoulis,"Brownian Movement and Markov Processes," in Probability, Random Variable, and Stochastic Processes, 2nd ed. New York: McGraw-Hill, 1984, pp. 515-553.

18. T. Kreuz, J.S Haas, A. Morelli, H.D. Abarbanel, and A. Politi J. Neurosci. Meth. 165, 151-161(2007).

19. J.D. Victor and K.P Purpura, Network 8, 127-164 (1997)

20. J. Dauwels, F. Vialatte, T. Weber and A. Cichocki, Advanced in Neuro-Information Processing 5506, 177-185.

21. P. Rowat, Neural Comput. 19, 1215-1250 (2007).

22. J. Dauwels, F. Vialatte, T. Weber and A. Cichocki, Neural Comput. 21, 2152-2208 (2009).

23. M. S. Lewicki, Network 9, 53-78 (1998). 